\title{
Measuring the burden of childhood blindness
}

Globally, the prevalence of blindness among children is estimated to be approximately one tenth of that in adults, at around 0.7 per $1000 .{ }^{1-3}$ However, blindness in childhood has far reaching implications for the affected child and family, and throughout life profoundly influences educational, employment, personal, and social prospects. ${ }^{4}$ Thus, the control of childhood blindness has been identified as a priority of the World Health Organisation's (WHO) global initiative for the elimination of avoidable blindness by the year $2020 .^{5}$

Measures of disease frequency alone, however, afford a limited understanding of the public health significance of childhood blindness. The global financial cost of blindness with an onset during childhood, in terms of loss of earning capacity (per capita GNP), is greater than the cost of adult blindness and has recently been estimated to be between US $\$ 6000$ million and $\$ 27000$ million. ${ }^{6}$ Most of this is accounted for by children living in high income countries, where the prevalence is less, but life expectancy and earning capacity greater, than in low income countries. These financial costs alone, however, provide only one perspective of the public health burden of blindness. Improved understanding and quantification require the application of indicators which measure the impact of blindness in terms of morbidity (years of disability suffered) as well as mortality (years of life lost through premature blindness associated death). Such indicators are useful in identifying those in the population in greatest need and for setting priorities in provision of health services. They are also important in the assessment of effectiveness of interventions and their economic evaluation. Finally, they offer a means of prioritising the agenda for future research.

The use of composite measures of morbidity and mortality, together with epidemiological and demographic indicators of the burden of disease, is well known in international public health. However, there is considerable disagreement about the most appropriate measures and the methods used to derive them. ${ }^{7}$ Indeed, some have advised that the use of these composite measures be reconsidered on the basis that health status and burden of disease cannot be expressed in a single figure..$^{9-12}$ An attempt has been made to measure the burden of visual impairment and blindness in adults in the Global Burden of Disease Study, ${ }^{713}$ which has attracted attention recently. The primary indicator of burden of disease, specifically developed for this study, was the time based outcome measure of disability adjusted life years (DALY) ${ }^{8}$ which is similar to the quality adjusted life year (QALY). ${ }^{14}$ Blindness was categorised, by expert consensus, in the penultimate class of increasingly severe disability. ${ }^{13}$ Cataract, glaucoma, trachoma, and onchocerciasis were implicated, either directly or as risk factors for other disorders, in $3.1 \%$ of deaths worldwide and were estimated to result in $1.9 \%$ of global DALYs lost. ${ }^{7}$ By comparison, diabetes mellitus, implicated in $5.5 \%$ of deaths, accounted for the same percentage of DALYs. ${ }^{7}$ The potential for estimating the burden of childhood blindness from this study is limited by the categorisation of the major causes of blindness in children within other groups of disorders such as malnutrition, communicable diseases, perinatal disorders, and congenital anomalies. Nevertheless, the cost effective- ness of some strategies to reduce child mortality which also have implications for childhood blindness has already been demonstrated: vitamin A supplementation, at about $\$ 1$ per DALY saved, continues to rank as one of the most cost effective of all health interventions. ${ }^{14}$

The four general concepts behind the development of the DALY were: firstly, all health outcomes which affect social welfare should be included in any indicator of health status; secondly, of the personal characteristics of the affected individual, only age and sex are to be considered in the calculation; thirdly, like health outcomes should be treated as like, irrespective of the characteristics of the community in which the affected individual lives; and fourthly, time should be the unit of measurement. ${ }^{8}$ While the rationale behind these principles is broadly accepted, there is debate about the validity of the guiding assumptions as well as the weighting and formulas used in calculating this essentially economic indicator. ${ }^{9-12}$

In public health ophthalmology the use of measures of burden of disease has, so far, been limited. There is a need for debate and collaboration between epidemiologists, ophthalmic professionals, health economists, and social scientists to promote the development of indicators of the burden of visual impairment. Evans and Ranson have recently proposed the use of the handicap adjusted life year (HALY), ${ }^{16}$ a prevalence based indicator similar to the DALY, calculated by summing years of life lost because of premature mortality and the years of life lived with handicap (adjusted for its severity) with both discounted to reflect time preference-that is, the greater value of present than future years of life. ${ }^{15} 16$ They have used this indicator to estimate the global burden of trachomatous visual impairment ${ }^{15}$ and in an economic evaluation of a trachoma control programme. ${ }^{16}$ Their sensitivity analysis (that is, varying the value of the factors used in their calculations), showed that the range of HALYs lost attributable to trachomatous visual impairment was very wide. The estimate was particularly sensitive to the values of disease prevalence, the age structure of the visually impaired population, and the discount rate used. Therefore, the authors urged cautious interpretation of the calculated disease burden, with particular attention to the "impressive variability in the underlying assumptions on which the estimates are based" ${ }^{15}$ This work highlights the constraints to further development and refinement of such indicators imposed by the lack of complete information about visually impaired individuals.

Even if a single composite measure were desirable, in most countries the necessary data for estimating the burden of mortality and morbidity attributable to the causes of childhood blindness are not available. Data regarding adults, where these are available, are inapplicable. These gaps in our knowledge are significant and will need to be addressed if we are to improve our ability to measure the burden of childhood blindness effectively and accurately. Thus, there is a need for better population based estimates of the prevalence and incidence of different causes of blindness. Longitudinal studies are required to determine age at onset and the duration of disease by cause. The increased risk of death associated with blindness in childhood needs to be quantified in both developing and 
industrialised nations where the likely causes are so different. $^{2}$ The problem of defining and measuring the disability or handicap resulting from visual impairment in childhood is challenging and, among other approaches, it will be necessary to develop visual outcome measures, including quality of life, which can be used in children of different ages. The findings of these areas of research have wide implications for practice and policy.

Thus, with our present knowledge it is difficult to estimate, with any certainty, the burden of childhood blindness in terms of morbidity and mortality. As up to $70 \%$ of childhood blindness worldwide might be effectively avoided by currently available primary, secondary, and tertiary preventive strategies, ${ }^{25}$ the need to improve existing strategies, especially in developing countries, as well as to develop new strategies, particularly in industrialised countries, to control childhood blindness is clear. However, given the economic considerations in healthcare provision in both settings, there is a need to demonstrate the relative value of these strategies to overall public health. Furthermore, there is a need to assess their effectiveness through their impact on the burden of disease. Thus we suggest that measuring the burden of childhood blindness accurately, as well as developing ways of effectively comparing it with other causes of morbidity, remain important goals.

Jugnoo Rahi was supported by a Medical Research Council clinical training fellowship during the preparation of this paper.

No special funding.

No conflict of interest.

J S RAHI

Department of Epidemiology and Public Health and Department of Ophthalmology,

Institute of Child Health/Great Ormond Street Hospital and Institute of Ophthalmology, 30 Guilford Street, London WC1N 1EH
C E GILBERT

Department of Preventive Ophthalmology,

Institute of Ophthalmology, Bath Street,

London EC1V 9EI

A FOSTER

Department of Infectious and Tropical Diseases,

London School of Hygiene and Tropical Medicine,

30 Keppel Street, London WC1E 7HT

\section{MINASSIAN}

Department of Preventive Ophthalmology,

Institute of Ophthalmology, Bath Street,

London EC1V 9EI

1 Foster A, Gilbert C. Epidemiology of childhood blindness. Eye 1992;6:1736.

2 Foster A, Gilbert C. Epidemiology of visual impairment in children. In: Taylor D, ed. Paediatric ophthalmology. 2nd ed. London: Blackwell Science, 1997:3-12.

3 Thylefors B, Negrel A-D, Pararajasegaram R, et al. Global data on blindness. WHO/PBL/94.40. Geneva: Programme for the Prevention of Blindness, World Health Organisation, 1995.

4 Day S. Normal and abnormal visual development. In: Taylor D, ed. Paediatric ophthalmology. 2nd ed. London: Blackwell Science, 1997:13-28.

5 Thylefors B. A global initiative for the elimination of avoidable blindness. Am f Ophthalmol 1998;125:90-3.

6 Smith AF, Smith JG. The economic burden of global blindness: a price too high! Br f Ophthalmol 1996;80:276-7.

7 Murray CJL, Lopez AD. Global mortality, disability, and the contribution of risk factors: Global Burden of Disease Study. Lancet 1997;349:1436-42.

8 Murray CJL. Quantifying the burden of disease: the technical basis for disability-adjusted life years. Bull World Health Organ 1994;72:429-45.

9 Robine JM. Measuring the burden of disease. Lancet 1998;352:758-9.
10 Murray CJL, Lopez AD. The utility of DALYS for public health policy and Murray CJL, Lopez AD. The utility of DALYS for public healt
research: a reply. Bull World Health Organ 1997;75:377-81.

11 Sayers B McA, Fliedner TM. The critique of DALYs: a counter-reply. Bull World Health Organ 1997;75:383-4.

12 DALY Review Group. DALY review, AHCR News. Bull World Health Organ 1997;74:333-4.

13 Murray CJL, Lopez AD. Regional patterns of disability-free life expectancy and disability-adjusted life expectancy: Global Burden of Disease Study. Lancet 1997;349:1347-52.

14 World Bank. The World development report 1993: investing in health. New York: Oxford University Press, 1993

15 Evans GE, Ranson ME. The global burden of trachomatous visual impairment. II: assessing burden. Int Ophthalmol 1995;19:271-80.

16 Evans GE, Ranson ME, Kyaw TA, et al. Cost effectiveness and cost utility of preventing trachomatous visual impairment: lessons from 30 years of trachoma control in Burma. Br f Ophthalmol 1996;80:880-9. 